\title{
The Upshot of Up States in the Neocortex: From Slow Oscillations to Memory Formation
}

\author{
Kari L. Hoffman, ${ }^{1}$ Francesco P. Battaglia, ${ }^{2 *}$ Kenneth Harris, ${ }^{3 *}$ Jason N. MacLean, ${ }^{4 \star}$ Lisa Marshall,,${ }^{5 *}$ and \\ Mayank R. Mehta ${ }^{6 *}$ \\ ${ }^{1}$ Department of Psychology, York University, Toronto, Ontario, Canada M3J 1P3, ${ }^{2}$ Swammerdam Institute for Life Sciences-Animal Physiology and Cognitive \\ Neuroscience, Universiteit van Amsterdam, 1098 SM Amsterdam, The Netherlands, ${ }^{3}$ Center for Molecular and Behavioral Neuroscience, Rutgers, The State \\ University of New Jersey, Newark, New Jersey 07102, ${ }^{4}$ Department of Neurobiology, University of Chicago, Chicago, Illinois 60637, ${ }^{5}$ Department of \\ Neuroendocrinology, University of Luebeck, 23538 Luebeck, Germany, and ${ }^{6}$ Department of Neuroscience, Brown University, Providence, Rhode Island 02912
}

Key words: memory consolidation; slow wave; non-REM; EEG; single unit; local field potential; calcium imaging; cell assembly; memory formation; plasticity; sleep; synchrony

A sleeping brain is by no means dormant: most cortical neurons, primarily detached from the influence of stimuli in the environment, are nevertheless active, just as they are during behavior. Although neural activity is preserved during sleep, the structure of the activity changes significantly, yielding bouts of widespread high-amplitude synchronized fluctuations in activity. These fluctuations, known as "slow oscillations" (Steriade et al., 1993), alternate between intervals of strong activity ("Up states") and intervals of almost complete silence ("Down states"), which spread coherently throughout the cortex at a typical frequency of $\sim 1 \mathrm{~Hz}$ during the sleep stage known as slow-wave sleep (SWS), as well as under certain types of anesthesia. The neocortical slow oscillation is thought to be generated intrinsically, depending on recurrent excitatory synaptic transmission (Sanchez-Vives and McCormick, 2000; Petersen et al., 2003).

The structure of slow oscillations may have implications for memory consolidation. Growing evidence supports the view that the transition of memories from a labile, hippocampaldependent state to a stable or consolidated, hippocampalindependent state depends on activity-driven plasticity processes that take place during sleep (Marr, 1971; Maquet, 2001; Dudai, 2004; Frankland and Bontempi, 2005), with a special role suggested for SWS (Buzsáki, 1989; Sejnowski and Destexhe, 2000). Evidence for this comes from the observation that patterns of hippocampal and neocortical activity elicited during a task appear to be replayed during SWS after the task. Although coordinated replay between hippocampus and neocortex could reflect

\footnotetext{
Received Aug. 1, 2007; revised Aug. 30, 2007; accepted Sept. 3, 2007.

This work was supported by the following: Fondation Fyssen, European Union Integrated Projects ICEA, BACS, and NeuroProbes (F.P.B.); Kavli Institute for Brain Science, National Eye Institute, National Institute of Neurological Disorders and Stroke, and Human Frontiers Science Project (J.N.M.); the Max Planck Society and the Canadian Foundation for Innovation Leaders Opportunity Fund (K.L.H.); the Sloan Foundation and National Institutes of Health (NIH) Grant R01MH073245 (K.H.); Deutsche Forschungsgemeinschaft grant Sonderforschungsbereich 654 "Plasticity and Sleep" (L.M.); a career award from the National Science Foundation, a Young Scholar Award from National Alliance for Research on Schizophrenia and Depression, and a grant from NIH-Collaborative Research in Computational Neuroscience (M.R.M.).

*F.P.B., K.H., J.N.M., L.M., and M.R.M. contributed equally to this work and are presented in alphabetical order. Correspondence should be addressed to Kari L. Hoffman, York University, 4700 Keele Street, Toronto, Ontario, Canada M3J 1P3. E-mail: khoffman@yorku.ca.

DOI:10.1523/JNEUROSCI.3501-07.2007

Copyright $\odot 2007$ Society for Neuroscience $\quad 0270-6474 / 07 / 2711838-04 \$ 15.00 / 0$
}

one mechanism underlying memory consolidation, there is little evidence linking the neural phenomenon of replay with the behavioral phenomenon of memory consolidation.

The strongest evidence for this connection comes indirectly, through features of slow oscillations, including (1) resistance to interference from sensory inputs, (2) replaying or reprocessing of memory traces, and (3) widespread synchronization of neocortical activity and coordination with hippocampal activity. In addition, evidence of a causal relationship between slow oscillations and memory formation is emerging. In what follows, we will consider each of these points, in turn, presenting recent advances in our understanding of the architecture of slow oscillations and how they could facilitate memory consolidation.

\section{Going “off-line”}

Although slow oscillations occur throughout the neocortex, its relationship to activity produced by the thalamus or sensory inputs remains unclear. Using mouse somatosensory thalamocortical slices and calcium imaging, thalamic inputs were observed to trigger network slow-oscillation Up states, which repeatedly involved a particular population of neurons activated in a particular sequence. These evoked Up states were statistically indistinguishable in the numbers, identities, and sequences of cells activated from spontaneous Up state activations (MacLean et al., 2005). Furthermore, it was the cortex itself that determined the specifics of the repeatable activations observed in both spontaneous and evoked Up states. Although the thalamus can trigger Up states, it appears to be ineffective at driving the neocortex during Up states: thalamic stimulation during ongoing Up states failed to perturb activity within individual neurons as well as within the active ensemble. Neither spontaneous nor thalamically evoked Up states were grossly altered when the thalamus is stimulated as they are occurring. Furthermore, spiking responses to stimulation were hampered, i.e., they failed to summate as expected from additional thalamic stimulation. In contrast to the insensitive neocortical response during Up states, stimulation during Down states strongly engaged neocortical networks (Petersen et al., 2003). Thus, the repeatable sequential activation dynamics in slices during Up states are apparently held "off-line" from sensory inputs by the Up state itself. Indeed, the persistence of the 
dynamics observed in these network synchronizations suggests that these dynamics may represent a solution to avoiding interference from incoming sensory signals during periods of memory replay.

\section{Neocortical patterns during Up states}

These observations suggest that, in slices, Up states are accompanied by stereotyped sequential activity patterns within the neurons of a local cortical column. However, is the same true in vivo? Cortical spontaneous activity in vivo can show a bewildering variety of patterns, from traveling waves (Petersen et al., 2003; Massimini et al., 2004) to oscillations such as sleep spindles; however, it appears that the slow oscillation organizes many of these other patterns (Steriade et al., 1993).

The observation of ensembles of single neurons provides a more detailed view of this phenomenon, beyond the global dynamical process. The structure of spiking activity in cortical populations during slow-oscillation Up states in vivo was investigated in a recent study in rat neocortex, using multisite silicon microelectrodes (Luczak et al., 2007). Transitions from Down to Up states were accompanied by a stable, sequential firing pattern, with the majority of neurons ( $~ 90 \%)$ exhibiting a unique temporal profile as the Up state progressed. At the start of the sequence, spike times were controlled with up to millisecond precision, but as the sequence progressed, timing accuracy decayed in a scalar manner similar to that seen in behavioral timing tasks (Meck, 2003). As in human scalp recordings, Up states spread as traveling waves, but the activity sequence in a local population was consistent regardless of direction of wave propagation, suggesting that in vivo as well as in vitro, the arrival of an Up state to a cortical column initiates a stereotyped pattern of information flow through the local cortical population. Thus, in principle, patterned activity during Up states could be generated in local populations, independently of activity in other brain structures, such as the thalamus. In practice, the hippocampus, a structure associated with memory formation, interacts reciprocally with the neocortex during Up states.

\section{The beat of a different drum}

According to some theories of memory consolidation (Marr, 1971; Buzsáki, 1989; Squire, 1992; McClelland et al., 1995), memories are thought to be minted rapidly in the hippocampus during behavior and transferred to the neocortex during slow-wave sleep for long-term storage. Evidence for the role of the hippocampus in rapid memory formation comes from the observation that, over repeated traversals of a location, hippocampal place cells produce a backward "anticipatory" expansion of location-specific firing and a marked increase in firing rate (Mehta and McNaughton, 1997; Mehta et al., 1997, 2002; Mehta and Wilson, 2000). Computational models suggest that the NMDA- and spike timing-dependent plasticity of synapses could mediate this rapid experience-dependent place cell plasticity (Blum and Abbott, 1996; Mehta and Wilson, 2000; Mehta et al., 2000, 2002).

For these experience-dependent changes in the hippocampus to affect neocortical memory traces, the two structures must interact. Hippocampal activity during SWS is characterized by the presence of "sharp waves," short (50-150 ms) bursts of activation that punctuate an otherwise relatively silent background. Hippocampal memory traces of recent behavioral experiences are replayed not only during SWS (Wilson and McNaughton, 1994) but specifically during sharp waves that occur within SWS (Kudrimoti et al., 1999). Moreover, it has been suggested that the cortical oscillations during SWS and hippocampal sharp-wave events are coupled (Sirota et al., 2003; Battaglia et al., 2004). This could provide a mechanism whereby the hippocampal reactivation could trigger activation of neocortical memory traces to be consolidated. Conversely, cortical Up states have been found to precede hippocampal sharp waves (Hahn et al., 2006, 2007; Isomura et al., 2006; Mölle et al., 2006; Ji and Wilson, 2007), which does not support the hypothesis that the hippocampus drives the neocortex during SWS. Implications of these observations for consolidation need to be explored in more detail (Mehta, 2007).

Less is known about the reactivation of memory traces in the neocortex, although periods of reactivation appear to be coordinated between the neocortical and hippocampal neural populations (Qin et al., 1997; Ji and Wilson, 2007), and this hippocampal reactivation may serve to coordinate memory traces that are widely distributed throughout the neocortex (Hoffman and McNaughton, 2002). An important prerequisite for this type of interaction is that the appropriate neural ensembles or "memory traces" are involved in these interactions, as suggested by the recent observations that (1) groups of cells that coactivated during the preceding experience (Battaglia et al., 2007) reactivate in discrete bouts during Up states and related spindle $(7-12 \mathrm{~Hz})$ oscillations, respecting the sequential order of activation (Ji and Wilson, 2007), and (2) the hippocampus influences the same subgroup of cells in the rat prefrontal cortex both during sleep and awake behavior (Peyrache et al., 2007).

These studies focused on the role of principal neurons in corticohippocampal interactions. The contribution of inhibition to this process was investigated using whole-cell recordings from the hippocampus in vivo, revealing that the hippocampal interneurons showed the same slow-wave oscillations as neocortical neurons (Hahn et al., 2006). The slow-wave modulation of hippocampal interneurons was weaker than cortical slow-wave modulation and followed cortical activity, suggesting that cortical activity may drive hippocampal interneurons. Furthermore, the granule cells of the dentate gyrus showed slow-wave modulation similar to the CA1 interneurons, whereas the CA1 pyramidal neurons did not show any modulation and were weakly hyperpolarized during cortical Up states (Hahn et al., 2007). These whole-cell recording studies suggest that neocortical activity could drive hippocampal activity during SWS, as part of a process that erases recent memory traces from the hippocampus (Mehta, 2007), while transferring them to the neocortex. If so, slow oscillations should lead to stronger neocortical memory traces, facilitating recall of the associated memory.

\section{Linking memory formation and slow-wave oscillations}

The neuronal interactions described above suggest how slow waves may be involved in processes of memory consolidation, without directly testing the impact on memory per se. Recently, one study revealed that exposure to a previously learned cue during SWS produced increased hippocampal blood oxygenation level-dependent activity during SWS and better recall after awakening (Rasch et al., 2007). Additional studies in humans showed that slow waves could be facilitated through electric (Marshall et al., 2006a,b) and magnetic (Massimini et al., 2007) stimulation. In both studies, the EEG power within the spindle frequency band was simultaneously increased. Sleep spindles have been associated with previous learning as well as synaptic plasticity and subsequent memory performance (Rosanova and Ulrich, 2005; Born et al., 2006; Eschenko et al., 2006). The ability of slowoscillation stimulation to enhance sleep spindle activity indicates that a functional neurophysiological system is set into motion. 
Underlining this concept, electric slow-oscillation stimulation also had an impact at the behavioral level (Marshall et al., 2006b). Compared with evening performance before sleep, memory for verbal and nonverbal declarative (hippocampus-dependent) tasks was boosted by slow-oscillation stimulation applied shortly after the onset of stage 2 sleep. Memory enhancement was specific for the slow-wave frequency band, because otherwise comparable stimulation at theta frequency did not improve memory.

An interesting implication of slow-oscillation stimulation is that an extracellular field potential is itself sufficient to modify cognitive processing. Despite the clear shift between Up and Down states in single cells, the cortical slow-wave oscillation has been characterized as a network mechanism (Volgushev et al., 2006). Along the same line, the synchronizing effect of induced electric fields was also found stronger for neuronal networks than for single neurons (Francis et al., 2003; Bikson et al., 2004; Deans et al., 2007). Thus, understanding how slow-oscillation stimulation translates into central nervous activity may even be of broader significance in establishing a comprehensive picture of CNS processing.

\section{Summary and future challenges}

Here, we described some of the characteristics of neural population activity during slow-wave sleep that may facilitate memory formation. The independence of the neocortex from external inputs and its interdependence with hippocampal activity sets the stage for memory reprocessing. Activity patterns in the hippocampus stand as a model for how neocortical populations might also form memory traces, and, indeed, there is evidence that distributed neocortical populations can spontaneously replay during sleep based on previously elicited activity patterns. Finally, a causal link between slow-wave oscillations and memory formation has been demonstrated.

The collective set of results from these studies indicates associations between slow oscillations, hippocampal-neocortical interactions, and memory formation, yet there is much left to be understood. Across the diversity of techniques and species used to determine the relationship between hippocampal and neocortical activity, the specific interactions vary in direction and sleep stage (Ribeiro and Nicolelis, 2004). Additional converging lines of evidence will be needed before the mechanisms of hippocampal-neocortical interactions can be understood.

Among the greatest gaps in this regard involves the scale of recording and species used. Very little data lie between the levels of analysis that include human scalp EEG (or functional magnetic resonance imaging) and the single-unit ensemble recordings in rat or intracellular recordings in cat, rat, and mouse slice. Measurements at the mesoscopic scale, as with local field potentials, collected in parallel with macroscopic scalp EEG and microscopic single units mark one important bridge. In a similar vein, the comparative approach is underexploited. For example, very few studies relate sleep and the neural traces of memories in the nonhuman primate (but see Hoffman and McNaughton, 2002). Many monkey species form rich representations of their fluctuating social environment, making them well suited for studying the microscopic to macroscopic neural processes that enable episodic memory formation.

Finally, cutting across species and level of observation, a key question that remains to be addressed is "what are the computational operations performed in the hippocampus and neocortex during sleep?" Although the transfer of information from the hippocampus to the neocortex could be an important component, it is probably not the only factor. Some evidence suggests that SWS may erase hippocampal memory traces (Mehta, 2007), perhaps freeing up hippocampal resources once the neocortical reprocessing has been achieved. Erasure in the form of synaptic homeostasis has been proposed for neocortex as well (Tononi and Cirelli, 2006), although implications for consolidation are not yet clear. It is potentially significant that, with consolidation, memories change in nature (Nadel and Moscovitch, 1997), becoming less dependent on context (Winocur et al., 2007) and richer in schematic, abstract structure (Tse et al., 2007). How these processes are implemented by brain circuits is still not well understood, but the converging lines of research highlighted here suggest that the answers are within reach.

\section{References}

Battaglia FP, Sutherland GR, McNaughton BL (2004) Hippocampal sharp wave bursts coincide with neocortical "up-state" transitions. Learn Mem 11:697-704.

Battaglia FP, Peyrache A, Benchenane K, Khamassi M, Douchamp V, Tierney PL, Wiener SI (2007) Time course of reactivation of memory-related cell ensembles in the rat medial prefrontal cortex during sleep. Soc Neurosci Abstr 33:427.4.

Bikson M, Inoue M, Akiyama H, Deans JK, Fox JE, Miyakawa H, Jefferys JG (2004) Effects of uniform extracellular DC electric fields on excitability in rat hippocampal slices in vitro. J Physiol (Lond) 557:175-190.

Blum KI, Abbott LF (1996) A model of spatial map formation in the hippocampus of the rat. Neural Comput 8:85-93.

Born J, Rasch B, Gais S (2006) Sleep to remember. The Neuroscientist $12: 410-424$.

Buzsáki G (1989) Two-stage model of memory trace formation: a role for "noisy" brain states. Neuroscience 31:551-570.

Deans JK, Powell AD, Jefferys JG (2007) Sensitivity of coherent oscillations in rat hippocampus to AC electric fields. J Physiol (Lond) 583:555-565.

Dudai Y (2004) The neurobiology of consolidations, or, how stable is the engram? Annu Rev Psychol 55:51-86.

Eschenko O, Mölle M, Born J, Sara SJ (2006) Elevated sleep spindle density after learning or after retrieval in rats. J Neurosci 26:12914-12920.

Francis JT, Gluckman BJ, Schiff SJ (2003) Sensitivity of neurons to weak electric fields. J Neurosci 23:7255-7261

Frankland PW, Bontempi B (2005) The organization of recent and remote memories. Nat Rev Neurosci 6:119-130.

Hahn TT, Sakmann B, Mehta MR (2006) Phase-locking of hippocampal interneurons' membrane potential to neocortical up-down states. Nat Neurosci 9:1359-1361.

Hahn TT, Sakmann B, Mehta MR (2007) Differential responses of hippocampal subfields to cortical up-down states. Proc Natl Acad Sci USA 104:5169-5174.

Hoffman KL, McNaughton BL (2002) Coordinated reactivation of distributed memory traces in primate neocortex. Science 297:2070-2073.

Isomura Y, Sirota A, Ozen S, Montgomery S, Mizuseki K, Henze DA, Buzsáki $G$ (2006) Integration and segregation of activity in entorhinalhippocampal subregions by neocortical slow oscillations. Neuron 52:871-882.

Ji D, Wilson MA (2007) Coordinated memory replay in the visual cortex and hippocampus during sleep. Nat Neurosci 10:100-107.

Kudrimoti HS, Barnes CA, McNaughton BL (1999) Reactivation of hippocampal cell assemblies: effects of behavioral state, experience, and EEG dynamics. J Neurosci 19:4090-4101.

Luczak A, Bartho P, Marguet SL, Buzsáki G, Harris KD (2007) Sequential structure of neocortical spontaneous activity in vivo. Proc Natl Acad Sci USA 104:347-352.

MacLean JN, Watson BO, Aaron GB, Yuste R (2005) Internal dynamics determine the cortical response to thalamic stimulation. Neuron 48:811-823.

Maquet P (2001) The role of sleep in learning and memory. Science 294:1048-1052.

Marr D (1971) Simple memory: a theory for archicortex. Philos Trans R Soc Lond B Biol Sci 262:23-81.

Marshall L, Mölle M, Born J (2006a) Oscillating current stimulation-slow oscillation stimulation during sleep. Retrieved October 15, 2007, from http:// www.natureprotocals.com/2006/11/09/oscillating_current_stimulatio.php. 
Marshall L, Helgadóttir H, Mölle M, Born J (2006b) Boosting slow oscillations during sleep potentiates memory. Nature 444:610-613.

Massimini M, Huber R, Ferrarelli F, Hill S, Tononi G (2004) The sleep slow oscillation as a traveling wave. J Neurosci 24:6862-6870.

Massimini M, Ferrarelli F, Esser SK, Riedner BA, Huber R, Murphy M, Peterson MJ, Tononi G (2007) Triggering sleep slow waves by transcranial magnetic stimulation. Proc Natl Acad Sci USA 104:8496-8501.

McClelland JL, McNaughton BL, O’Reilly RC (1995) Why there are complementary learning systems in the hippocampus and neocortex: insights from the successes and failures of connectionist models of learning and memory. Psychol Rev 102:419-457.

Meck WH (2003) Functional and neural mechanisms of interval timing. Boca Raton, FL: CRC.

Mehta MR (2007) Cortico-hippocampal interaction during up-down states and memory consolidation. Nat Neurosci 10:13-15.

Mehta MR, McNaughton BL (1997) Expansion and shift of hippocampal place fields: evidence for synaptic potentiation during behavior. In: Computational neuroscience: trends in research (Bower J, ed), pp 741-745. New York: Plenum.

Mehta MR, Wilson MA (2000) From hippocampus to V1: effect of LTP on spatio-temporal dynamics of receptive fields. Neurocomputing 32:905-911.

Mehta MR, Barnes CA, McNaughton BL (1997) Experience-dependent, asymmetric expansion of hippocampal place fields. Proc Natl Acad Sci USA 94:8918-8921.

Mehta MR, Quirk MC, Wilson MA (2000) Experience-dependent asymmetric shape of hippocampal receptive fields. Neuron 25:707-715.

Mehta MR, Lee AK, Wilson MA (2002) Role of experience and oscillations in transforming a rate code into a temporal code. Nature 417:741-746.

Mölle M, Yeshenko O, Marshall L, Sara SJ, Born J (2006) Hippocampal sharp wave-ripples linked to slow oscillations in rat slow-wave sleep. J Neurophysiol 96:62-70.

Nadel L, Moscovitch M (1997) Memory consolidation, retrograde amnesia and the hippocampal complex. Curr Opin Neurobiol 7:217-227.

Petersen CC, Hahn TT, Mehta M, Grinvald A, Sakmann B (2003) Interaction of sensory responses with spontaneous depolarization in layer $2 / 3$ barrel cortex. Proc Natl Acad Sci USA 100:13638-13643.

Peyrache A, Benchenane K, Khamassi M, Douchamp V, Tierney PL, Battaglia FP, Wiener SI (2007) Rat medial prefrontal cortex neurons are modu- lated by both hippocampal theta rhythm and sharp wave-ripple events. Soc Neurosci Abstr 33:427.3.

Qin YL, McNaughton BL, Skaggs WE, Barnes CA (1997) Memory reprocessing in corticocortical and hippocampocortical neuronal ensembles. Philos Trans R Soc Lond B Biol Sci 352:1525-1533.

Rasch B, Buchel C, Gais S, Born J (2007) Odor cues during slow-wave sleep prompt declarative memory consolidation. Science 315:1426-1429.

Ribeiro S, Nicolelis MA (2004) Reverberation, storage, and postsynaptic propagation of memories during sleep. Learn Mem 11:686-696.

Rosanova M, Ulrich D (2005) Pattern-specific associative long-term potentiation induced by a sleep spindle-related spike train. J Neurosci 25:9398-9405.

Sanchez-Vives MV, McCormick DA (2000) Cellular and network mechanisms of rhythmic recurrent activity in neocortex. Nat Neurosci 3:1027-1034.

Sejnowski TJ, Destexhe A (2000) Why do we sleep? Brain Res 886:208-223.

Sirota A, Csicsvari J, Buhl D, Buzsáki G (2003) Communication between neocortex and hippocampus during sleep in rodents. Proc Natl Acad Sci USA 100:2065-2069.

Squire LR (1992) Memory and the hippocampus: a synthesis from findings with rats, monkeys, and humans. Psychol Rev 99:195-231.

Steriade M, Nunez A, Amzica F (1993) A novel slow ( $<1 \mathrm{~Hz}$ ) oscillation of neocortical neurons in vivo: depolarizing and hyperpolarizing components. J Neurosci 13:3252-3265.

Tononi G, Cirelli C (2006) Sleep function and synaptic homeostasis. Sleep Med Rev 10:49-62.

Tse D, Langston RF, Kakeyama M, Bethus I, Spooner PA, Wood ER, Witter MP, Morris RG (2007) Schemas and memory consolidation. Science 316:76-82.

Volgushev M, Chauvette S, Mukovski M, Timofeev I (2006) Precise longrange synchronization of activity and silence in neocortical neurons during slow-wave oscillations. J Neurosci [Erratum (2006) 26:table of contents] 26:5665-5672.

Wilson MA, McNaughton BL (1994) Reactivation of hippocampal ensemble memories during sleep. Science 265:676-679.

Winocur G, Moscovitch M, Sekeres M (2007) Memory consolidation or transformation: context manipulation and hippocampal representations of memory. Nat Neurosci 10:555-557. 